\title{
On cross-diffusion effects on flow over a vertical surface using a linearization method
}

\author{
Precious Sibanda", Ahmed Abdalmgid Khidir and Faiz Gadelmola Awad
}

\author{
* Correspondence: sibandap@ukzn. \\ ac.za \\ School of Mathematics, Statistics \& \\ Computer Science, University of \\ KwaZulu-Natal, Private Bag X01, \\ Scottsville, Pietermaritzburg 3209, \\ South Africa
}

\begin{abstract}
In this article, we explore the use of a non-perturbation linearization method to solve the coupled highly nonlinear system of equations due to flow over a vertical surface subject to a magnetic field. The linearization method is used in combination with an asymptotic expansion technique. The effects of Dufour, Soret and magnetic filed parameters are investigated. The velocity, temperature and concentration distributions as well as the skin-friction, heat and mass transfer coefficients have been obtained and discussed for various physical parametric values. The accuracy of the solutions has been tested using a local non-similarity method. The results show that the non-perturbation technique is an accurate numerical algorithm that converges rapidly and may serve as a viable alternative to finite difference and finite element methods for solving nonlinear boundary value problems.
\end{abstract}

Mathematics Subject Classification (2000): 76D05; 74S30; 76E06; 76M25.

Keywords: cross-diffusion, free convection, linearization method, incompressible flow, magneto-hydrodynamics (MHD)

\section{Introduction}

Convection driven by density variations caused by two different components which have different rates of diffusion plays an important role in fluid dynamics since such flows occur naturally in many physical and engineering processes. Heat and salt in sea water provide perhaps the best known example of double-diffusive convection, Stern [1]. Other examples of double diffusive convection are encountered in diverse applications such as in chemical and petroleum industries, filtration processes, food processing, geophysics and in the modeling of solar ponds and magma chambers. A review of the literature in this subject can be found in Nield and Bejan [2].

One of the earliest studies of double diffusive convection was by Nield [3]. Baines and Gill [4] investigated linear stability boundaries, while Rudraiah et al. [5] used the nonlinear perturbation theory to investigate the onset of double diffusive convection in a horizontal porous layer. Poulikakos [6] presented the linear stability analysis of thermosolutal convection using the Darcy-Brinkman model. Bejan and Khair [7] presented a multiple scale analysis of heat and mass transfer about a vertical plate embedded in a porous medium. They considered concentration gradients which aid or oppose thermal gradients. Related studies on double diffusive convection have been undertaken by, among others, Lai [8], Afify [9], and Makinde and Sibanda [10].

(c) 2012 Sibanda et al; licensee Springer. This is an Open Access article distributed under the terms of the Creative Commons Attribution License (http://creativecommons.org/licenses/by/2.0), which permits unrestricted use, distribution, and reproduction in any medium, provided the original work is properly cited. 
Investigations by, among others, Eckert and Drake [11] and Mortimer and Eyring [12] have provided examples of flows such as in the geosciences, where diffusionthermo and thermal-diffusion effects are quite significant. Anjalidevi and Devi [13] showed that diffusion-thermo and thermal-diffusion effects are significant when density differences exist in the flow regime. In general, Diffusion-thermo and thermal-diffusion effects have been found to be particularly important for intermediate molecular weight gases in binary systems that are often encountered in chemical engineering processes. Theoretical studies of the Soret and Dufour effects on double diffusive convection have been made by many researchers, among them, Kafoussias and Williams [14], Postelnicu [15], Mansour et al. [16], Narayana and Sibanda [17], and Awad et al. [18].

In this article, we investigate convective heat and mass transfer along a vertical flat plate in the presence of diffusion-thermo, thermal diffusion effects, and an external magnetic field. The governing momentum, heat and mass transfer equations are, in general, strongly coupled and highly nonlinear. Apart from numerical methods, a number of semi-analytical techniques have in recent years been proposed to find approximate solutions of nonlinear boundary value problems. Some of these techniques, such as the Adomian decomposition method, the variational iteration method, the homotopy analysis method, the homotopy perturbation method, the differential transform method, etc, are now well known and their strengths and weaknesses well understood. Some of these weaknesses include, for example, small regions of convergence and the use of artificially inserted parameters (Liao [19], Geng [20]). The recent spectral homotopy analysis method (see Motsa et el. [21,22]) sought to improve the accuracy and efficiency of the homotopy analysis method while retaining its essence. The use of spectral methods also provided greater flexibility in the choice of basis functions. Nonetheless, the challenge to find more accurate, robust and computationally efficient solution techniques for nonlinear problems in engineering and science still remains.

A recent method that remains to be generalized and whose robustness remains to be tested in the case of highly nonlinear equations with a strong coupling is the successive linearization method (Makukula et al. [23,24]). This method has been used in a limited number of studies by, for example, Awad et al. [25]) and Motsa et al. [26] to solve fluid flow problems. In this study the coupled set of differential equations that describe convective heat and mass transfer flow along a vertical flat plate in the presence of diffusion-thermo, thermal diffusion effects and an external magnetic field are solved using the successive linearization method. A non-similarity technique is used to validate the linearization method.

\section{Problem formulation}

We consider the problem of double diffusive convection along a vertical plate with an external magnetic field imposed along the $y$-axis. The induced magnetic field is assumed to be negligible. The fluid temperature and solute concentration in the ambient fluid are $T_{\infty}, C_{\infty}$ and those at the surface are $T_{w}$ and $C_{w}$, respectively. The coordinates system and the flow configuration are shown in Figure 1.

Under the usual boundary layer and Boussinesq approximations the governing equations for a viscous incompressible fluid may be written as (Kafoussias and Williams [14]); 


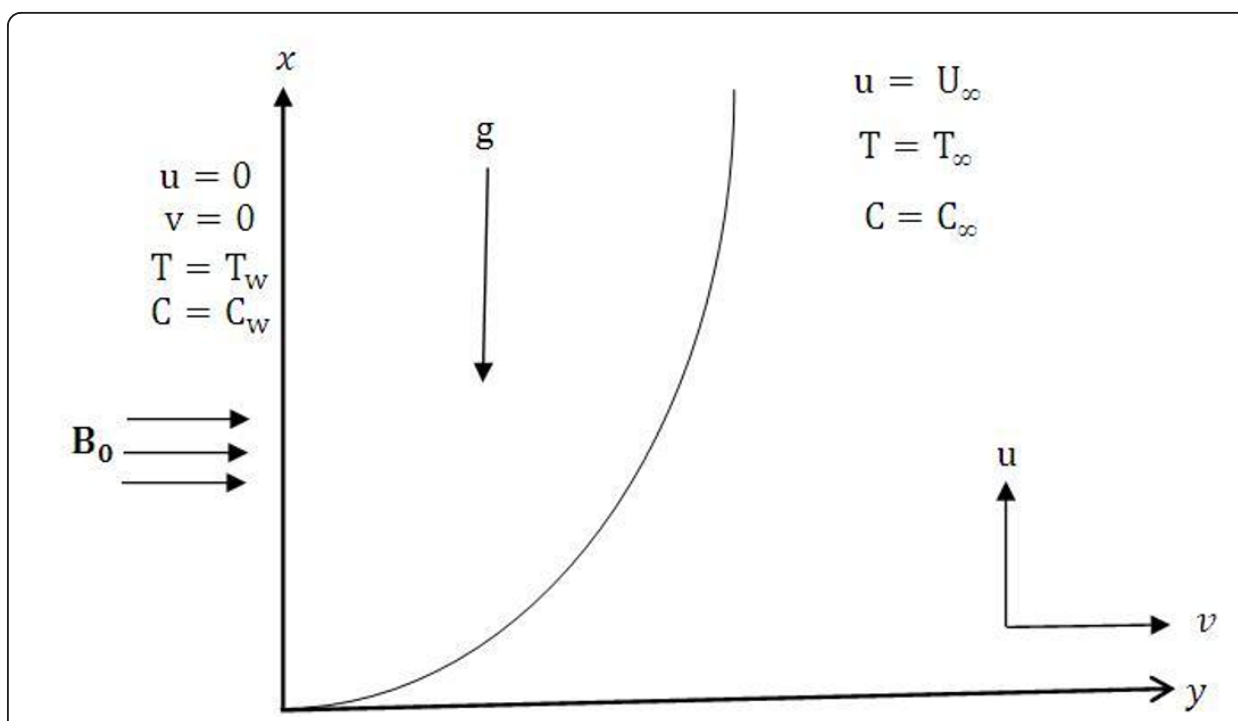

Figure 1 Physical model and coordinate system

$$
\begin{aligned}
& \frac{\partial u}{\partial x}+\frac{\partial v}{\partial y}=0 \\
& u \frac{\partial u}{\partial x}+v \frac{\partial u}{\partial y}=v \frac{\partial^{2} u}{\partial y^{2}}-\frac{\sigma B_{0}^{2}}{\rho} u+\mathrm{g} \beta_{T}\left(T-T_{\infty}\right)+\mathrm{g} \beta_{C}\left(C-C_{\infty}\right) \\
& u \frac{\partial T}{\partial x}+v \frac{\partial T}{\partial y}=\alpha \frac{\partial^{2} T}{\partial y^{2}}+\frac{D_{m} k_{T}}{c_{s} c_{p}} \frac{\partial^{2} C}{\partial y^{2}} \\
& u \frac{\partial C}{\partial x}+v \frac{\partial C}{\partial y}=D_{m} \frac{\partial^{2} C}{\partial y^{2}}+\frac{D_{m} k_{T}}{T_{m}} \frac{\partial^{2} T}{\partial y^{2}}
\end{aligned}
$$

subject to the boundary conditions

$$
\begin{aligned}
& u=0, \quad v=0, \quad T=T_{w}, \quad C=C_{w} \text { on } y=0, \\
& u=U_{\infty}, \quad T=T_{\infty}, \quad C=C_{\infty} \text { when } y \rightarrow \infty .
\end{aligned}
$$

where $u$ and $v$ are the velocity components along the $x$ - and $y$-axes, respectively $T$ and $C$ are the fluid temperature and solute concentration across the boundary layer, $v$ is the kinematic viscosity, $\rho$ is the fluid density, $\sigma$ is the electrical conductivity, $B_{0}$ is the uniform magnetic field, $\beta_{T}$ and $\beta_{C}$ are the coefficients of thermal and solutal expansions, $D_{m}$ is the thermal diffusivity, $k_{T}$ is the thermal diffusion ratio, $c_{s}$ is the concentration susceptibility, $c_{p}$ is the fluid specific heat capacity, $T_{m}$ is the mean fluid temperature, $U_{\infty}$ is the free stream velocity and $\mathrm{g}$ is the gravitational acceleration.

To satisfy the continuity equation (1), we define the stream function $\psi$ in terms of the velocity by

$$
u=\frac{\partial \psi}{\partial y} \quad \text { and } \quad v=-\frac{\partial \psi}{\partial x}
$$


and introduce the following dimensionless variables

$$
\xi=\frac{x}{\ell}, \eta=\left(\frac{U_{\infty}}{\nu \xi}\right)^{1 / 2} \gamma, \quad \psi=\left(U_{\infty} \nu \xi\right)^{1 / 2} f(\xi, \eta), \quad \theta(\xi, \eta)=\frac{T-T_{\infty}}{T_{w}-T_{\infty}}, \quad \phi(\xi, \eta)=\frac{C-C_{\infty}}{C_{w}-C_{\infty}},
$$

where, without loss of generality, we take the constant length $\ell$ to be unity in the subsequent analysis. Using equations (7) in (2)-(4), we get the transformed equations

$$
\begin{aligned}
& f^{\prime \prime \prime}+\frac{1}{2} f f^{\prime \prime}-H a_{x} f^{\prime}+G r_{x} \theta+G c_{x} \phi=\xi\left(f^{\prime} \frac{\partial f^{\prime}}{\partial \xi}-f^{\prime \prime} \frac{\partial f}{\partial \xi}\right), \\
& \frac{1}{\operatorname{Pr}} \theta^{\prime \prime}+\frac{1}{2} f \theta^{\prime}+D_{f} \phi^{\prime \prime}=\xi\left(f^{\prime} \frac{\partial \theta}{\partial \xi}-\theta^{\prime} \frac{\partial f}{\partial \xi}\right), \\
& \frac{1}{S c} \phi^{\prime \prime}+\frac{1}{2} f \phi^{\prime}+S_{r} \theta^{\prime \prime}=\xi\left(f^{\prime} \frac{\partial \phi}{\partial \xi}-\phi^{\prime} \frac{\partial f}{\partial \xi}\right),
\end{aligned}
$$

with corresponding boundary conditions

$$
\left.\begin{array}{l}
f=0, \quad f^{\prime}=0, \quad \theta=1, \quad \phi=1, \quad \text { on } \quad \eta=0, \\
f^{\prime}=1, \quad \theta=0, \quad \phi=0, \quad \text { when } \quad \eta \rightarrow \infty .
\end{array}\right\}
$$

The fluid and physical parameters in equations (8)-(10) are the local thermal and solutal Grashof numbers $G r_{x}$ and $G c_{x}$, the local magnetic field parameter $H a_{x}$, the Prandtl number $P r$, the Dufour number $D_{f}$, the Soret number $S_{r}$, and the Schmidt number $S c$. These parameters are defined in equations (12), (13) below.

$$
\begin{aligned}
& G r_{x}=\frac{\mathrm{g} \beta_{T}\left(T_{w}-T_{\infty}\right) x}{v^{2}}, \quad G c_{x}=\frac{\mathrm{g} \beta_{C}\left(C_{w}-C_{\infty}\right) x}{v^{2}}, \quad H a_{x}=\frac{\sigma B_{0}^{2} x}{\rho U_{\infty}}, \quad \operatorname{Pr}=\frac{v}{\alpha}, \\
& D_{f}=\frac{D_{m} K_{T}\left(C_{w}-C_{\infty}\right)}{\alpha C_{s} C_{p}\left(T_{w}-T_{\infty}\right)}, \quad S_{r}=\frac{K_{T} D_{m}\left(T_{w}-T_{\infty}\right)}{\alpha T_{m}\left(C_{w}-C_{\infty}\right)}, \quad S c=\frac{v}{D_{m}} .
\end{aligned}
$$

The parameters of engineering interest in heat and mass transport problems are the skin friction coefficient $C_{f x}$, the Nusselt number $N u_{x}$ and the Sherwood number $S h_{x}$. These parameters characterize the surface drag, the wall heat and mass transfer rates, respectively, and are defined by

$$
\begin{aligned}
& C_{f x}=\frac{\mu}{\rho U^{2}}\left(\frac{\partial u}{\partial y}\right)_{y=0}=\frac{f^{\prime \prime}(\xi, 0)}{\sqrt{R e_{x}}}, \\
& N u_{x}=\frac{-x}{T_{w}-T_{\infty}}\left(\frac{\partial T}{\partial y}\right)_{y=0}=-\sqrt{\operatorname{Re}_{x}} \theta^{\prime}(\xi, 0),
\end{aligned}
$$

and

$$
S h_{x}=\frac{-x}{C_{w}-C_{\infty}}\left(\frac{\partial C}{\partial y}\right)_{y=0}=-\sqrt{R e_{x}} \phi^{\prime}(\xi, 0),
$$

where $R e_{x}=U_{\infty} x / v$. 


\section{Method of solution}

The main challenge in using the linearization method as described in Makukula et al. $[23,24]$ is how to generalize the method so as to find solutions of partial differential equations of the form (8)-(10). It is certainly not clear how the method may be applied directly to the terms on the right hand side of equations (8)-(10). For this reason, equations (8)-(10) are first simplified and reduced to sets of ordinary differential equations by assuming regular perturbation expansions for $f, \theta$, and $\varphi$ in powers of $\xi$ (which is assumed to be small) as follows

$$
f=f_{M_{1}}(\eta, \xi)=\sum_{i=0}^{M_{1}} \xi^{i} f_{i}(\eta), \quad \theta=\theta_{M_{1}}(\eta, \xi)=\sum_{i=0}^{M_{1}} \xi^{i} \theta_{i}(\eta), \quad \phi=\phi_{M_{1}}(\eta, \xi)=\sum_{i=0}^{M_{1}} \xi^{i} \phi_{i}(\eta)
$$

where $M_{1}$ is the order of the approximate solution. Substituting (17) into equations (8)-(10) and equating the coefficients of like powers of $\xi$, we obtain the zeroth order set of ordinary differential equations

$$
\begin{aligned}
& f_{0}^{\prime \prime \prime}+\frac{1}{2} f_{0} f_{0}^{\prime \prime}-H a_{x} f_{0}^{\prime}+G r_{x} \theta_{0}+G c_{x} \phi_{0}=0, \\
& \frac{1}{\operatorname{Pr}} \theta_{0}^{\prime \prime}+\frac{1}{2} f_{0} \theta_{0}^{\prime}+D_{f} \phi_{0}^{\prime \prime}=0, \\
& \frac{1}{S c} \phi_{0}^{\prime \prime}+\frac{1}{2} f_{0} \phi_{0}^{\prime}+S_{r} \theta_{0}^{\prime \prime}=0,
\end{aligned}
$$

with corresponding boundary conditions

$$
\left.\begin{array}{ll}
f_{0}=0, f_{0}^{\prime}=0, \theta_{0}=1, \phi_{0}=1, & \text { at } \eta=0 \\
f_{0}^{\prime}=1, \theta_{0}=0, \phi_{0}=0, & \text { as } \eta=\infty .
\end{array}\right\}
$$

The $O\left(\xi^{1}\right)$ equations are

$$
\begin{aligned}
& f_{1}^{\prime \prime \prime}+\frac{1}{2} f_{0} f_{1}^{\prime \prime}-\left(H a_{x}+f_{0}^{\prime}\right) f_{1}^{\prime}+\frac{3}{2} f_{0}^{\prime \prime} f_{1}+G r_{x} \theta_{1}+G c_{x} \phi_{1}=0, \\
& \frac{1}{\operatorname{Pr}} \theta_{1}^{\prime \prime}+\frac{1}{2} f_{0} \theta_{1}^{\prime}-f_{0}^{\prime} \theta_{1}+\frac{3}{2} \theta_{0}^{\prime} f_{1}+D_{f} \phi_{1}^{\prime \prime}=0, \\
& \frac{1}{S c} \phi_{1}^{\prime \prime}+\frac{1}{2} f_{0} \phi_{1}^{\prime}-f_{0}^{\prime} \phi_{1}+\frac{3}{2} \phi_{0}^{\prime} f_{1}+S_{r} \theta_{1}^{\prime \prime}=0,
\end{aligned}
$$

with boundary conditions

$$
\left.\begin{array}{ll}
f_{1}=0, f_{0}^{\prime}=0, \theta_{1}=0, \phi_{1}=0, & \text { at } \eta=0 \\
f_{1}^{\prime}=0, \theta_{1}=0, \phi_{1}=0, & \text { as } \eta=\infty .
\end{array}\right\}
$$

Finally, the $O\left(\xi^{2}\right)$ equations are

$$
\begin{aligned}
& f_{2}^{\prime \prime \prime}+\frac{1}{2} f_{0} f_{2}^{\prime \prime}-\left(H a_{x}+2 f_{0}^{\prime}\right) f_{2}^{\prime}+\frac{5}{2} f_{0}^{\prime \prime} f_{2}+G r_{x} \theta_{2}+G c_{x} \phi_{2}=f_{1}^{\prime} f_{1}^{\prime}-\frac{3}{2} f_{1} f_{1}^{\prime \prime}, \\
& \frac{1}{\operatorname{Pr}} \theta_{2}^{\prime \prime}+\frac{1}{2} f_{0} \theta_{2}^{\prime}-2 f_{0}^{\prime} \theta_{2}+\frac{5}{2} \theta_{0}^{\prime} f_{2}+D_{f} \phi_{2}^{\prime \prime}=f_{0}^{\prime} \theta_{1}-\frac{3}{2} f_{1} \theta_{1}^{\prime}
\end{aligned}
$$




$$
\frac{1}{S c} \phi_{2}^{\prime \prime}+\frac{1}{2} f_{0} \phi_{2}^{\prime}-2 f_{0}^{\prime} \phi_{2}+\frac{5}{2} \phi_{0}^{\prime} f_{2}+S_{r} \theta_{2}^{\prime \prime}=f_{1}^{\prime} \phi_{1}-\frac{3}{2} f_{1} \phi_{1}^{\prime} .
$$

These equations have to be solved subject to boundary conditions

$$
\left.\begin{array}{ll}
f_{2}=0, f_{2}^{\prime}=0, \theta_{2}=0, \phi_{2}=0, & \text { at } \eta=0 \\
f_{2}^{\prime}=0, \theta_{2}=0, \phi_{2}=0, & \text { as } \eta=\infty .
\end{array}\right\}
$$

The coupled system of equations (18)-(20), (22)-(24), and (26)-(28) together with the associated boundary conditions (21), (25), and (29) may be solved independently pairwise one after another. These equations may now be solved using the successive linearization method in the manner described in Makukula et al. $[23,24])$. We begin by solving equations (18)-(20) with boundary conditions (21).

The successive linearization method is a non-perturbation method requiring neither the presence of an embedded perturbation parameter nor the addition of an artificial parameter. The method is therefore free of the major limitations associated with other perturbation methods. In the SLM algorithm assumption is made that the functions $f_{0}$ $(\eta), \theta_{0}(\eta)$, and $\varphi_{0}(\eta)$ may be expressed as

$$
f_{0}(\eta)=F_{i}(\eta)+\sum_{m=0}^{i-1} F_{m}(\eta), \quad \theta_{0}(\eta)=\Theta_{i}(\eta)+\sum_{m=0}^{i-1} \Theta_{m}(\eta), \quad \phi(\eta)=\Phi_{i}(\eta)+\sum_{m=0}^{i-1} \Phi_{m}(\eta),
$$

where $F_{i}, \Theta_{i}$, and $\Phi_{i}(i \geq 1)$ are unknown functions and $F_{m}, \Theta_{m}$, and $\Phi_{m}$ are successive approximations which are obtained by recursively solving the linear part of the system that is obtained from substituting equations (30) in (18)-(20). In choosing the form of the expansions (30), prior knowledge of the general nature of the solutions, as is often the case with perturbation methods, is not necessary.

Suitable initial guesses $F_{0}(\eta), \Theta_{0}(\eta)$, and $\Phi_{0}(\eta)$ which are selected to satisfy the boundary conditions (21) are

$$
F_{0}(\eta)=\eta+e^{-\eta}-1, \quad \Theta_{0}(\eta)=e^{-\eta}, \quad \Phi_{0}(\eta)=e^{-\eta},
$$

The subsequent solutions $F_{i}, \Theta_{i}$, and $\Phi_{i}$ are obtained by iteratively solving the linearized form of the equations that are obtained by substituting equation (30) in the governing equations (18)-(20). The linearized equations to be solved are

$$
\begin{aligned}
& F_{i}^{\prime \prime \prime}+a_{1, i-1} F_{i}^{\prime \prime}-H a_{x} F_{i}^{\prime}+a_{2, i-1} F_{i}+G r_{x} \Theta_{i}+G c_{x} \Phi_{i}=r_{1, i-1} \\
& \frac{1}{\operatorname{Pr}} \Theta_{i}^{\prime \prime}+b_{1, i-1} \Theta_{i}^{\prime}+b_{2, i-1} F_{i}+D_{f} \Phi_{i}^{\prime \prime}=r_{2, i-1}, \\
& \frac{1}{S c} \Phi_{i}^{\prime \prime}+c_{1, i-1} \Phi_{i}^{\prime}+c_{2, i-1} F_{i}+\operatorname{Sr} \Theta_{i}^{\prime \prime}=r_{3, i-1},
\end{aligned}
$$

subject to the boundary conditions

$$
F_{i}(0)=F_{i}^{\prime}(0)=F_{i}^{\prime}(\infty)=\Theta_{i}(0)=\Theta_{i}(\infty)=\Phi_{i}(0)=\Phi_{i}(\infty),
$$


where the coefficients parameters $a_{k, i-1}, b_{k, i-1}, c_{k, i-1}, d_{k, i-1}$, and $r_{k, i-1}$ are defined by

$$
\begin{aligned}
& a_{1, i-1}=\frac{1}{2} \sum_{m=0}^{i-1} F_{m}, \quad a_{2, i-1}=\frac{1}{2} \sum_{m=0}^{i-1} F_{m}^{\prime \prime}, \quad b_{1, i-1}=\frac{1}{2} \sum_{m=0}^{i-1} F_{m} \\
& b_{2, i-1}=\frac{1}{2} \sum_{m=0}^{i-1} \Theta_{m}^{\prime} \quad c_{1, i-1}=\frac{1}{2} \sum_{m=0}^{i-1} F_{m} \quad c_{2, i-1}=\frac{1}{2} \sum_{m=0}^{i-1} \Phi_{m^{\prime}}^{\prime} \\
& r_{1, i-1}=H a_{x} \sum_{m=0}^{i-1} F_{m}^{\prime}-\frac{1}{2} \sum_{m=0}^{i-1} F_{m} \sum_{m=0}^{i-1} F_{m}^{\prime \prime}-\sum_{m=0}^{i-1} F_{m}^{\prime \prime \prime}-G r_{x} \sum_{m=0}^{i-1} \Theta_{m}-G c_{x} \sum_{m=0}^{i-1} \Phi_{m}, \\
& r_{2, i-1}=-\frac{1}{\operatorname{Pr}} \sum_{m=0}^{i-1} \Theta_{m}^{\prime \prime}-\frac{1}{2} \sum_{m=0}^{i-1} F_{m} \sum_{m=0}^{i-1} \Phi_{m}^{\prime}-D_{f} \sum_{m=0}^{i-1} \Phi_{m^{\prime}}^{\prime \prime} \\
& r_{3, i-1}=-\frac{1}{S c} \sum_{m=0}^{i-1} \Phi_{m}^{\prime \prime}-\frac{1}{2} \sum_{m=0}^{i-1} F_{m} \sum_{m=0}^{i-1} \Phi_{m}^{\prime}-S r \sum_{m=0}^{i-1} \Phi_{m^{\prime}}^{\prime \prime}
\end{aligned}
$$

Once each solution $F_{i}, \Theta_{i}$, and $\Phi_{i}(i \geq 1)$, has been found from iteratively solving equations (32)-(34), the approximate solutions for the system (18)-(20) are obtained as

$$
f_{0}(\eta) \approx \sum_{i=0}^{M_{2}} F_{i}(\eta), \quad \theta_{0}(\eta) \approx \sum_{i=0}^{M_{2}} \Theta_{i}(\eta), \quad \phi_{0}(\eta) \approx \sum_{i=0}^{M_{2}} \Phi_{i}(\eta),
$$

where $M_{2}$ is the order of the SLM approximations. In this study, we used the Chebyshev spectral collocation method to solve equations (32)-(34). The physical region $[0$, $\infty)$ is first transformed into the spectral domain $[-1,1]$ using the domain truncation technique in which the problem is solved on the interval $[0, L]$, where $L$ is a scaling parameter used to invoke the boundary condition at infinity. This is achieved by using the mapping

$$
\frac{\eta}{L}=\frac{\varsigma+1}{2}, \quad-1 \leq \varsigma \leq 1,
$$

We discretise the spectral domain $[-1,1]$ using the Gauss-Lobatto collocation points given by

$$
\varsigma_{j}=\cos \frac{\pi j}{N}, \quad j=0,1, \ldots, N,
$$

where $N$ is the number of collocation points used. The unknown functions $F_{i}, \Theta_{i}$, and $\Phi_{i}$ are approximated at the collocation points by

$$
F_{i}(\varsigma) \approx \sum_{k=0}^{N} F_{i}\left(\varsigma_{k}\right) T_{k}\left(\varsigma_{j}\right), \quad \Theta_{i}(\varsigma) \approx \sum_{k=0}^{N} \Theta_{i}\left(\varsigma_{k}\right) T_{k}\left(\varsigma_{j}\right) \Phi_{i}(\varsigma) \approx \sum_{k=0}^{N} \Phi_{i}\left(\varsigma_{k}\right) T_{k}\left(\varsigma_{j}\right), j=0,1, \ldots, N
$$

where $T_{k}$ is the $k$ th Chebyshev polynomial defined as

$$
T_{k}(\varsigma)=\cos \left[k \cos ^{-1}(\varsigma)\right] \text {. }
$$

The derivatives of the variables at the collocation points are represented as

$$
F_{i}^{(r)}=\sum_{k=0}^{N} \mathbf{D}_{k j}^{r} F_{i}\left(\varsigma_{k}\right), \quad \Theta_{i}^{(r)}=\sum_{k=0}^{N} \mathbf{D}_{k j}^{r} \Theta_{i}\left(\varsigma_{k}\right), \quad \Phi_{i}^{(r)}=\sum_{k=0}^{N} \mathbf{D}_{k j}^{r} \Phi_{i}\left(\varsigma_{k}\right), \quad j=0,1, \ldots, N
$$


where $r$ is the order of differentiation and $\mathbf{D}=\frac{2}{L} \mathcal{D}$ with $\mathcal{D}$ being the Chebyshev spectral differentiation matrix whose entries are defined as;

$$
\left\{\begin{array}{l}
\mathcal{D}_{j k}=\frac{c_{j}(-1)^{j+1}}{c_{k} \zeta_{j}-\varsigma_{k}} \quad j \neq k ; j, k=0,1, \ldots, N, \\
\mathcal{D}_{k k}=-\frac{\zeta_{k}}{2\left(1-\varsigma_{k}^{2}\right)} \quad k=1,2, \ldots, N-1, \\
\mathcal{D}_{00}=\frac{2 N^{2}+1}{6}=-\mathcal{D}_{N N} .
\end{array}\right.
$$

Substituting equations (37)-(41) into (32)-(34) gives the following linear system of equations

$$
\mathbf{A}_{i-1} \mathbf{X}_{i}=\mathbf{R}_{i-1}
$$

subject to the boundary conditions

$$
F_{i}\left(\varsigma_{0}\right)=\sum_{k=0}^{N} \mathcal{D}_{0 k} F_{i}\left(\varsigma_{k}\right)=g_{i}\left(\varsigma_{0}\right)=\Theta_{i}\left(\varsigma_{0}\right)=\Phi_{i}\left(\varsigma_{0}\right)=F_{i}\left(\varsigma_{N}\right)=g_{i}\left(\varsigma_{N}\right)=\Theta_{i}\left(\varsigma_{N}\right)=\Phi_{i}\left(\varsigma_{N}\right)=0 .
$$

Here $\mathbf{A}_{i-1}$ is a $3(N+1) \times 3(N+1)$ square matrix, while $\mathbf{X}_{i}$ and $\mathbf{R}_{i-1}$ are $3(N+1) \times 1$ column vectors defined by

$$
\mathbf{A}_{i-1}=\left[\begin{array}{lll}
A_{11} & A_{12} & A_{13} \\
A_{21} & A_{22} & A_{23} \\
A_{31} & A_{32} & A_{33}
\end{array}\right], \quad \mathbf{X}_{i}=\left[\begin{array}{c}
\tilde{\mathbf{F}}_{i} \\
\tilde{\Theta}_{i} \\
\tilde{\Phi}_{i}
\end{array}\right], \quad \mathbf{R}_{i-1}=\left[\begin{array}{c}
\mathbf{r}_{1, i-1} \\
\mathbf{r}_{2, i-1} \\
\mathbf{r}_{3, i-1}
\end{array}\right]
$$

where

$$
\begin{aligned}
& \tilde{\mathbf{F}}_{i}=\left[F_{i}\left(\varsigma_{0}\right), F_{i}\left(\varsigma_{1}\right), \ldots, F_{i}\left(\varsigma_{N-1}\right), F_{i}\left(\varsigma_{N}\right)\right]^{T}, \\
& \tilde{\Theta}_{i}=\left[\Theta_{i}\left(\varsigma_{0}\right), \Theta_{i}\left(\varsigma_{1}\right), \ldots, \Theta_{i}\left(\varsigma_{N-1}\right), \Theta_{i}\left(\varsigma_{N}\right)\right]^{T}, \\
& \tilde{\Phi}_{i}=\left[\Phi_{i}\left(\varsigma_{0}\right), \Phi_{i}\left(\varsigma_{1}\right), \ldots, \Phi_{i}\left(\varsigma_{N-1}\right), \Phi_{i}\left(\varsigma_{N}\right)\right]^{T}, \\
& \mathbf{r}_{1, i-1}=\left[r_{1, i-1}\left(\varsigma_{0}\right), r_{1, i-1}\left(\varsigma_{1}\right), \ldots, r_{1, i-1}\left(\varsigma_{N-1}\right), r_{1, i-1}\left(\varsigma_{N}\right)\right]^{T} \\
& \mathbf{r}_{2, i-1}=\left[r_{2, i-1}\left(\varsigma_{0}\right), r_{2, i-1}\left(\varsigma_{1}\right), \ldots, r_{2, i-1}\left(\varsigma_{N-1}\right), r_{2, i-1}\left(\varsigma_{N}\right)\right]^{T} \\
& \mathbf{r}_{3, i-1}=\left[r_{3, i-1}\left(\varsigma_{0}\right), r_{3, i-1}\left(\varsigma_{1}\right), \ldots, r_{3, i-1}\left(\varsigma_{N-1}\right), r_{3, i-1}\left(\varsigma_{N}\right)\right]^{T}
\end{aligned}
$$

and

$$
\begin{aligned}
& A_{11}=\mathcal{D}^{3}+a_{1, i-1} \mathcal{D}^{2}-H a_{x} \mathcal{D}+\left[a_{3, i-1}\right], \quad A_{12}=\left[G r_{x}\right], \quad A_{13}=\left[G c_{x}\right], \\
& A_{21}=\left[b_{2, i-1}\right], \quad A_{22}=\operatorname{Pr}^{-1} \mathcal{D}^{2}+b_{1, i-1} \mathcal{D}, \quad A_{23}=D_{f} \mathcal{D}^{2} \quad A_{31}=\left[c_{2, i-1}\right], \\
& A_{32}=\operatorname{SrD}^{2}, \quad A_{33}=S c^{-1} \mathcal{D}^{2}+c_{1, i-1} \mathcal{D},
\end{aligned}
$$

where [.] is a diagonal matrix of size $(N+1) \times(N+1)$ and $a_{k, i-1}, b_{k, i-1}, c_{k, i-1}$ are diagonal matrices of size $(N+1) \times(N+1)$ and $T$ is the transpose. After modifying the matrix system (43) to incorporate the boundary conditions (44), the solution is obtained as

$$
\mathbf{X}_{i}=\mathbf{A}_{i-1}^{-1} \mathbf{R}_{i-1}
$$


Equation (53) gives a solution of (18)-(20) for $f_{0}, \theta_{0}$ and $\varphi_{0}$. The procedure is repeated to obtain the $O\left(\xi^{1}\right)$ and $O\left(\xi^{2}\right)$ solutions using equations (22)-(24) and (26)(28), respectively.

\section{Results and discussion}

In generating the results in this article, we determined through numerical experimentation that $L=15, N=40$, and $M_{2}=5$ gave sufficient accuracy for the linearization method. The value of the Prandtl number used is $P r=0.71$ which physically corresponds to air. The Schmidt number used $S c=0.22$ is for hydrogen at approximately $25^{\circ}$ and one atmospheric pressure (see Afify [27]). In concert with previous related studies, the Dufour and Soret numbers are chosen in such a way that their product is constant, provided the mean temperature $T_{m}$ is also kept constant.

To determine the accuracy and validate the linearization method, equations (8)-(10) were further solved using a local non-similarity method (LNSM) developed by Sparrow and $\mathrm{Yu}$ [28] and Sparrow et al. [29]. Previous studies have consistently used the Matlab bvp4c solver to evaluate the accuracy of the successive linearization method. However, as with other BVP solvers, the accuracy and convergence of the bvp4c algorithm depends on a good initial guess and works better for systems involving few equations (Shampine et al. [30]).

Tables 1, 2, and 3 show, firstly the effects of various parameters on the skin-friction and the local heat and mass transfer coefficients at different values of $\xi$ and, second, give a sense of the accuracy and convergence rate of the linearization method. The results from the two methods are in excellent agreement with the second order SLM series giving accuracy of up to five significant figures.

Table 1 shows the effect of increasing the magmatic field parameter $H a_{x}$ on the local skin friction, heat and mass transfer coefficients. We observe that increasing the magnetic field parameter reduces the local skin friction as well as the heat and mass transfer coefficients. In Table 2, we present the effect of the Soret parameter on $f^{\prime \prime}(0),-\theta^{\prime}(0)$,

Table 1 The effect of the magnetic field parameter $H a_{x}$ on $f^{\prime \prime}(0), \theta^{\prime}(0)$ and $\varphi^{\prime}(0)$ when $G r_{x}=0.5, G c_{x}=2, D_{f}=0.2$, and $S r=0.3$

\begin{tabular}{|c|c|c|c|c|c|c|c|}
\hline \multirow[b]{3}{*}{ Profile } & \multirow[b]{3}{*}{$H a_{x}$} & \multicolumn{3}{|c|}{$\xi=0.005$} & \multicolumn{3}{|c|}{$\xi=0.01$} \\
\hline & & \multicolumn{2}{|c|}{ SLM results } & \multirow[t]{2}{*}{ LNS Method } & \multicolumn{2}{|c|}{ SLM results } & \multirow[t]{2}{*}{ LNS Method } \\
\hline & & $M_{2}=2$ & $M_{2}=3$ & & $M_{2}=2$ & $M_{2}=3$ & \\
\hline & 0.0 & 3.045324 & 3.045324 & 3.045324 & 3.045324 & 3.045324 & 3.045324 \\
\hline & 0.5 & 2.299370 & 2.299370 & 2.299370 & 2.299370 & 2.299370 & 2.299370 \\
\hline \multirow[t]{5}{*}{$f^{\prime}(0)$} & 1.0 & 1.940291 & 1.940291 & 1.940291 & 1.940291 & 1.940291 & 1.940291 \\
\hline & 2.0 & 1.532041 & 1.532041 & 1.532041 & 1.532041 & 1.532041 & 1.532041 \\
\hline & 2.5 & 1.404033 & 1.404033 & 1.404033 & 1.404033 & 1.404033 & 1.404033 \\
\hline & 0.0 & 0.513493 & 0.513493 & 0.513493 & 0.513493 & 0.513493 & 0.513493 \\
\hline & 0.5 & 0.435640 & 0.435640 & 0.435640 & 0.435640 & 0.435640 & 0.435640 \\
\hline \multirow[t]{5}{*}{$-\theta^{\prime}(0)$} & 1.0 & 0.392869 & 0.392869 & 0.392869 & 0.392869 & 0.392869 & 0.392869 \\
\hline & 2.0 & 0.335307 & 0.335307 & 0.335307 & 0.335307 & 0.335307 & 0.335307 \\
\hline & 2.5 & 0.314405 & 0.314405 & 0.314405 & 0.314405 & 0.314405 & 0.314405 \\
\hline & 0.0 & 0.289548 & 0.289548 & 0.289548 & 0.289548 & 0.289548 & 0.289548 \\
\hline & 0.5 & 0.231673 & 0.231673 & 0.231673 & 0.231673 & 0.231673 & 0.231673 \\
\hline \multirow[t]{3}{*}{$-\varphi^{\prime}(0)$} & 1.0 & 0.202778 & 0.202778 & 0.202778 & 0.202778 & 0.202778 & 0.202778 \\
\hline & 2.0 & 0.169217 & 0.169217 & 0.169217 & 0.169217 & 0.169217 & 0.169217 \\
\hline & 2.5 & 0.157771 & 0.157771 & 0.157771 & 0.157771 & 0.157771 & 0.157771 \\
\hline
\end{tabular}


Table 2 The effect of the Soret parameter Sr on $f^{\prime \prime}(0), \theta^{\prime}(0)$ and $\varphi^{\prime}(0)$ when $G r_{x}=0.5, G c_{x}$ $=2$, and $H a_{x}=1$

\begin{tabular}{|c|c|c|c|c|c|c|c|}
\hline \multirow[b]{3}{*}{ Profile } & \multirow[b]{3}{*}{$S r$} & \multicolumn{3}{|c|}{$\xi=0.005$} & \multicolumn{3}{|c|}{$\xi=0.01$} \\
\hline & & \multicolumn{2}{|c|}{ SLM results } & \multirow[t]{2}{*}{ LNS Method } & \multicolumn{2}{|c|}{ SLM results } & \multirow[t]{2}{*}{ LNS Method } \\
\hline & & $M_{2}=2$ & $M_{2}=3$ & & $M_{2}=2$ & $M_{2}=3$ & \\
\hline & 0.1 & 1.933303 & 1.933303 & 1.933302 & 1.933303 & 1.933303 & 1.933302 \\
\hline & 0.4 & 1.946426 & 1.946426 & 1.946426 & 1.946426 & 1.946426 & 1.946426 \\
\hline \multirow[t]{5}{*}{$f^{\prime}(0)$} & 0.6 & 1.959632 & 1.959632 & 1.959632 & 1.959632 & 1.959632 & 1.959632 \\
\hline & 1.5 & 2.023004 & 2.023004 & 2.023004 & 2.023004 & 2.023004 & 2.023004 \\
\hline & 2.0 & 2.059313 & 2.059313 & 2.059313 & 2.059313 & 2.059313 & 2.059313 \\
\hline & 0.1 & 0.368159 & 0.368159 & 0.368159 & 0.368159 & 0.368159 & 0.368159 \\
\hline & 0.4 & 0.396905 & 0.396905 & 0.396905 & 0.396905 & 0.396905 & 0.396905 \\
\hline \multirow[t]{5}{*}{$-\theta^{\prime}(0)$} & 0.6 & 0.402187 & 0.402187 & 0.402187 & 0.402187 & 0.402187 & 0.402187 \\
\hline & 1.5 & 0.416430 & 0.416430 & 0.416430 & 0.416430 & 0.416430 & 0.416430 \\
\hline & 2.0 & 0.422788 & 0.422788 & 0.422788 & 0.422788 & 0.422788 & 0.422788 \\
\hline & 0.1 & 0.213565 & 0.213565 & 0.213565 & 0.213565 & 0.213565 & 0.213565 \\
\hline & 0.4 & 0.197708 & 0.197708 & 0.197708 & 0.197708 & 0.197708 & 0.197708 \\
\hline \multirow[t]{3}{*}{$-\varphi^{\prime}(0)$} & 0.6 & 0.187601 & 0.187601 & 0.187601 & 0.187601 & 0.187601 & 0.187601 \\
\hline & 1.5 & 0.141063 & 0.141063 & 0.141063 & 0.141063 & 0.141063 & 0.141063 \\
\hline & 2.0 & 0.114262 & 0.114262 & 0.114262 & 0.114262 & 0.114262 & 0.114262 \\
\hline
\end{tabular}

and $-\varphi^{\prime}(0)$ which are, respectively, proportional to the local skin friction coefficient, the local Nusselt number and Sherwood number. We observe that $f^{\prime \prime}(0)$ and $-\theta^{\prime}(0)$ increase with increases in $S r$, while $-\varphi^{\prime}(0)$ decreases as $S r$ increases. These results are confirmed in Table 3. Here the Nusselt number increases as the Soret number increases, while the opposite trend occurs as the Dufour number increases. The recent study by ElKabeir [31] shows that these results may be modified by injection, suction or the presence of a chemical reaction.

The influence of the various fluid and physical parameters on the fluid properties is given qualitatively in Figures 2, 3, 4, 5, 6 and 7. Figures 2 and 3 illustrate the effect of the magnetic filed parameter on the velocity $f(\eta)$, temperature $\theta$ and concentration $\varphi$ profiles within the boundary layer. We observe that, as expected, strengthening the magnetic field slows down the fluid motion due to an increasing drag force which acts against the flow if the magnetic field is applied in the normal direction. We also observe that the magnetic field parameter enhances the temperature and concentration profiles. The effect of broadening both the temperature and concentration distributions is to reduce the wall temperature and concentration gradients thereby reducing the heat and mass transfer rates at the wall.

Table 3 Soret and Dufour effects of the skin friction coefficient $C_{f}$, Nusselt number $\mathrm{Nu}$ and Sherwood number Sh when $G r_{x}=0.5, G c_{x}=2, S c=0.22$, and $H a_{x}=0.5$

\begin{tabular}{lllll}
\hline $\boldsymbol{S}_{\boldsymbol{r}}$ & $\boldsymbol{D}_{\boldsymbol{f}}$ & $\boldsymbol{C}_{\boldsymbol{f}}$ & $\boldsymbol{N u}$ & $\boldsymbol{S} \boldsymbol{h}$ \\
\hline 0.1 & 0.60 & 1.933303 & 0.368159 & 0.213565 \\
0.2 & 0.30 & 1.935047 & 0.386060 & 0.207941 \\
0.4 & 0.15 & 1.946426 & 0.396905 & 0.197708 \\
0.6 & 0.10 & 1.959632 & 0.402187 & 0.187601 \\
1.5 & 0.04 & 2.023004 & 0.416430 & 0.141063 \\
2.0 & 0.03 & 2.059313 & 0.422788 & 0.114262 \\
\hline
\end{tabular}




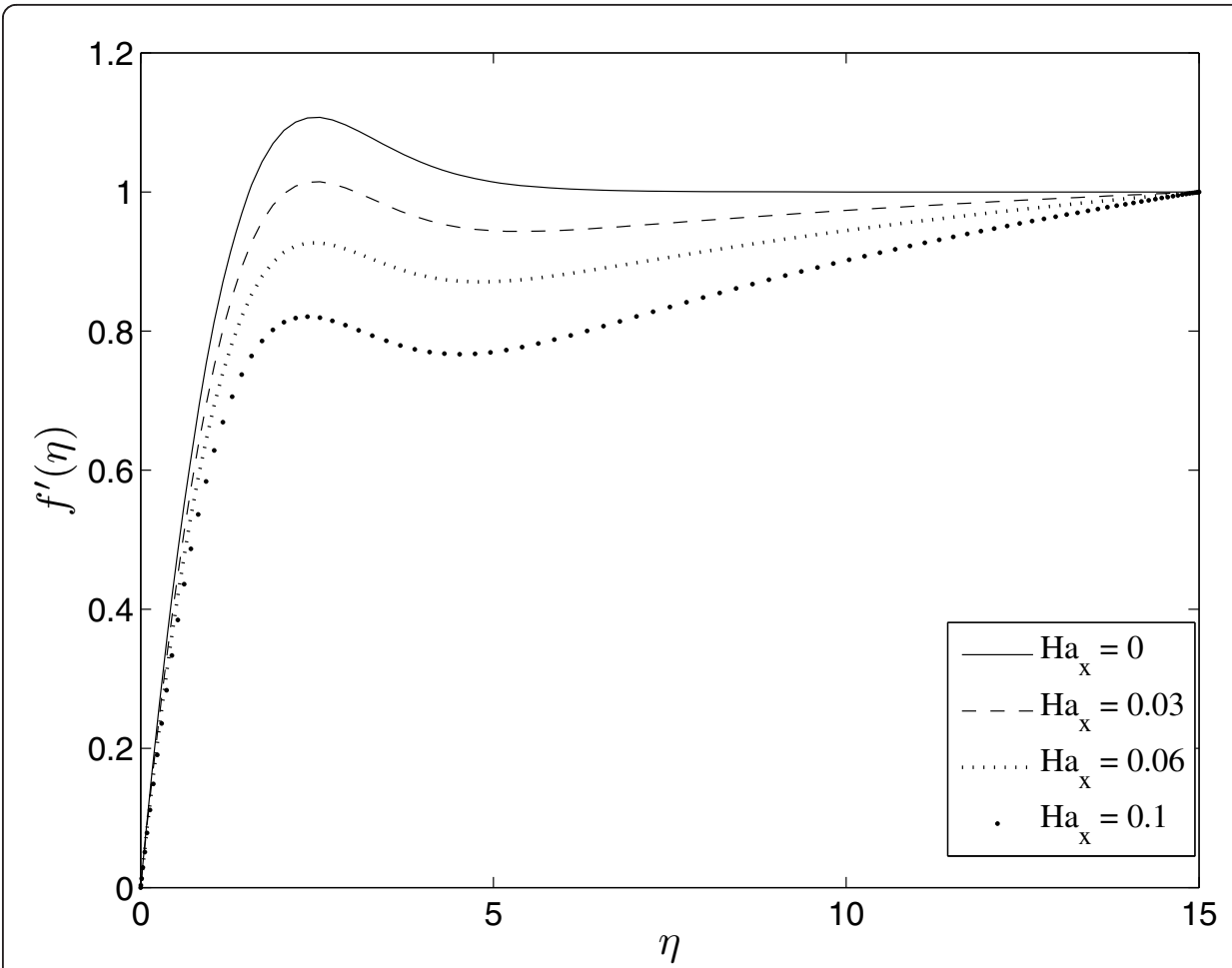

Figure 2 Effect of the magmatic field parameter $H a_{x}$ on $f^{\prime}(\eta)$ when $G r_{x}=0.5, G c_{x}=0.1, D_{f}=0.2, S r$ $=0.3$, and $\xi=0.01$.

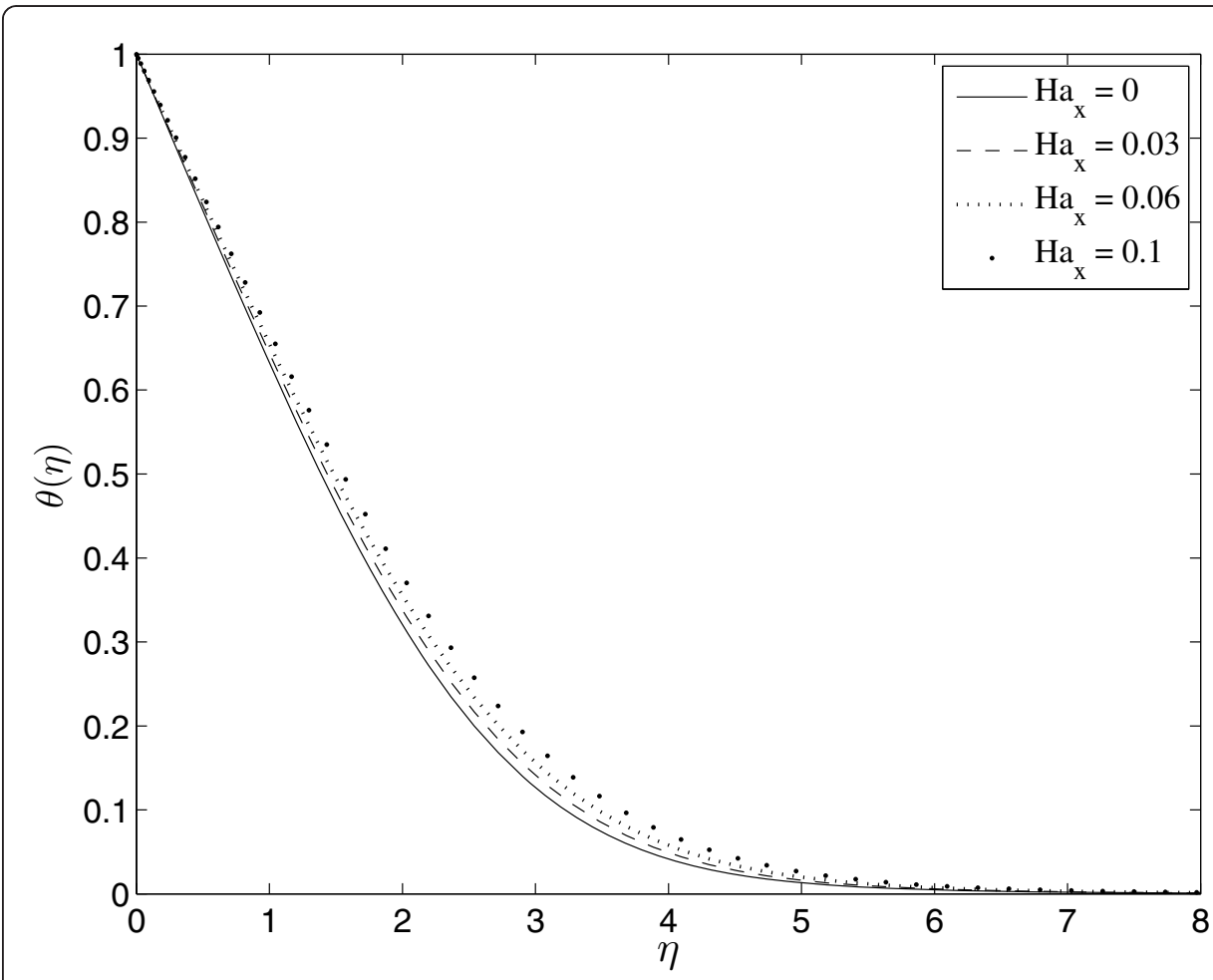

Figure 3 Effect of the magmatic field parameter $H a_{x}$ on $\theta(\eta)$ when $G r_{x}=0.5, G c_{x}=0.1, D_{f}=0.2, S r$ $=0.3$, and $\xi=0.01$. 


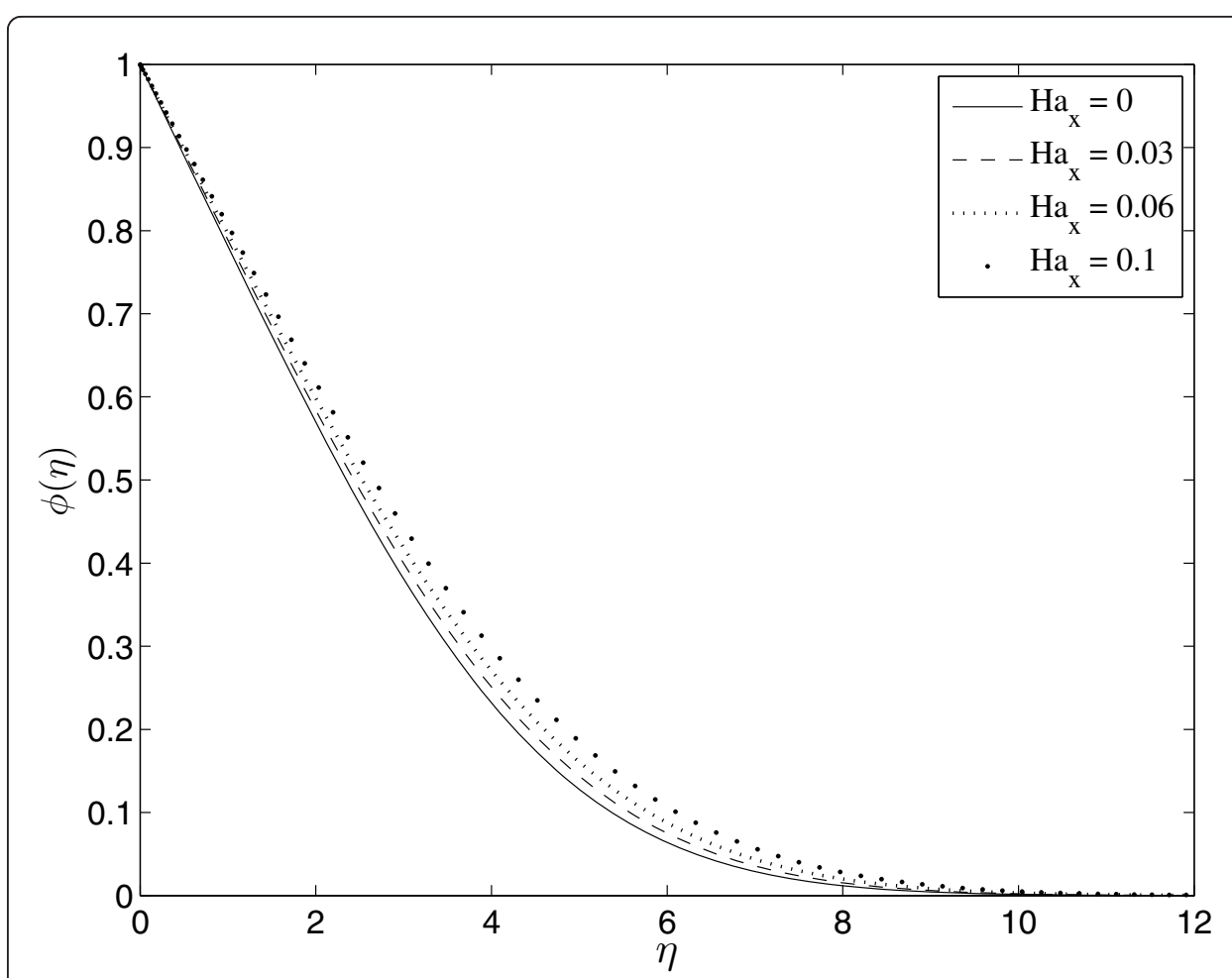

Figure 4 Effect of the magmatic field parameter $H a_{x}$ on $\varphi(\eta)$ when $G r_{x}=0.5, G c_{x}=0.1, D_{f}=0.2, S r$ $=0.3$, and $\xi=0.01$.

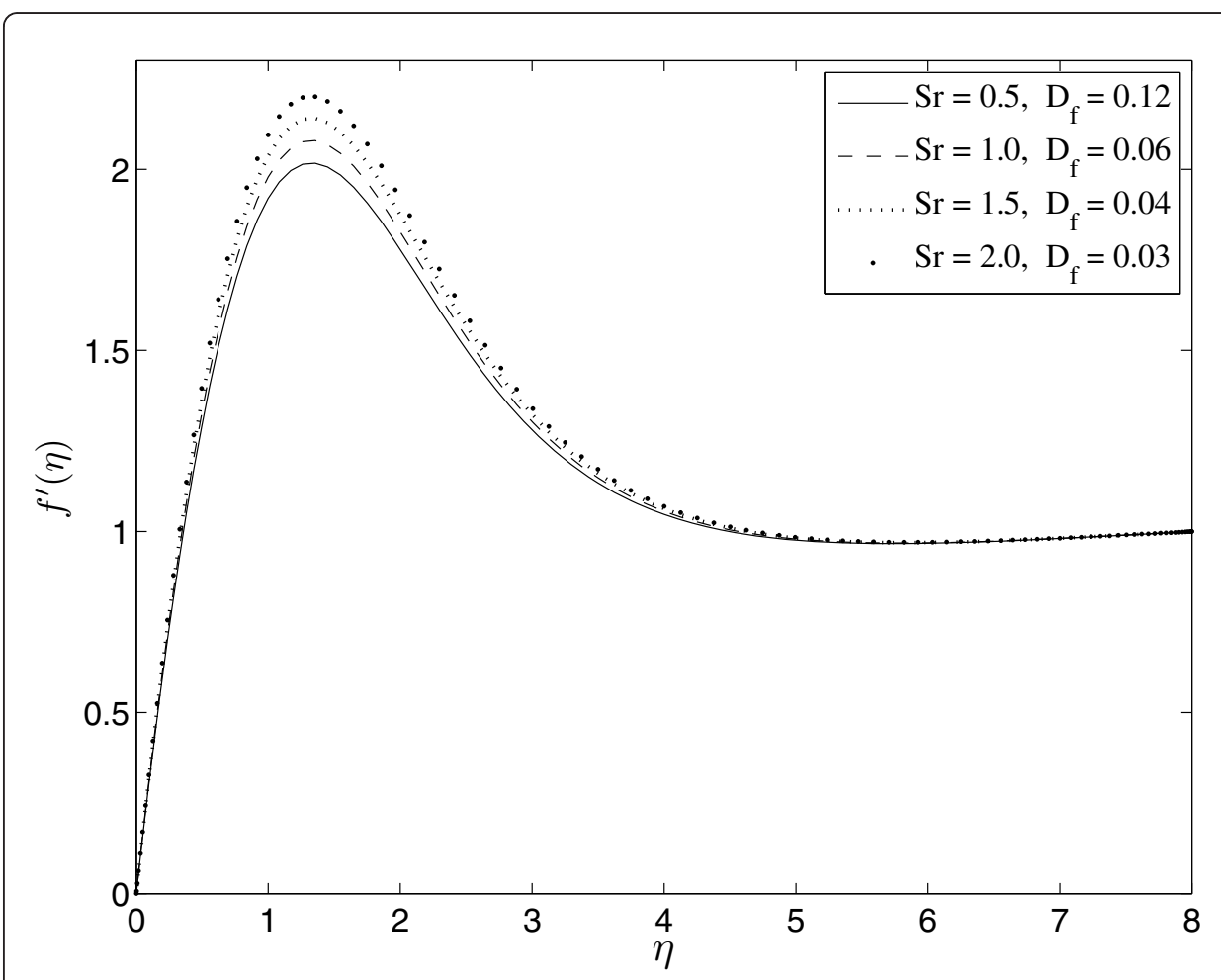

Figure 5 Effect of the Soret and Dufour parameters on the velocity $f^{\prime}(\eta)$ when $G r_{x}=0.5, G c_{x}=2.5$, $H a_{x}=0.1$, and $\xi=0.01$ 


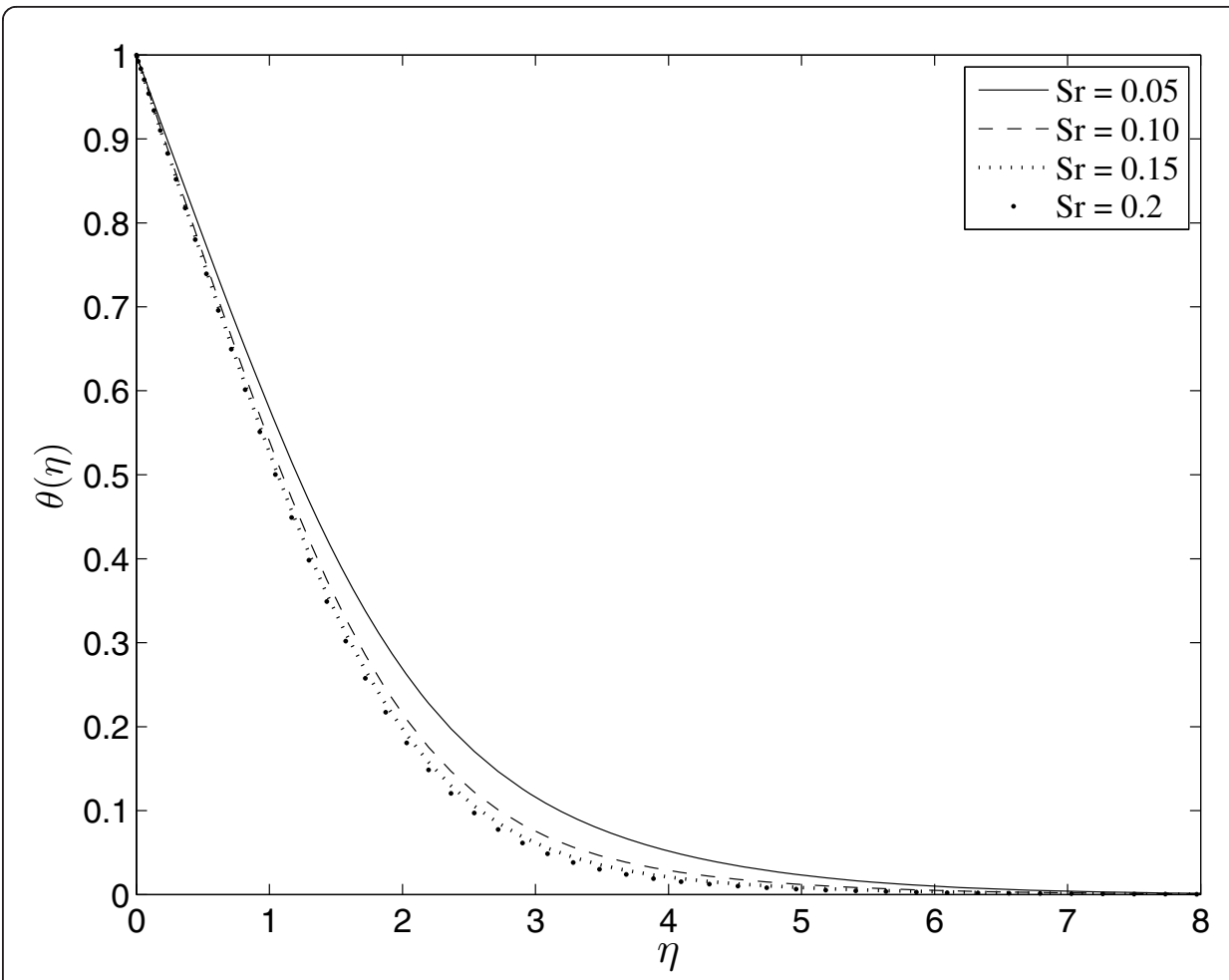

Figure 6 Effect of Soret parameter $S_{r}$ on $\theta(\eta)$ when $G r_{x}=0.1, G c_{x}=2.5, H a_{x}=0.1$, and $\xi=0.01$

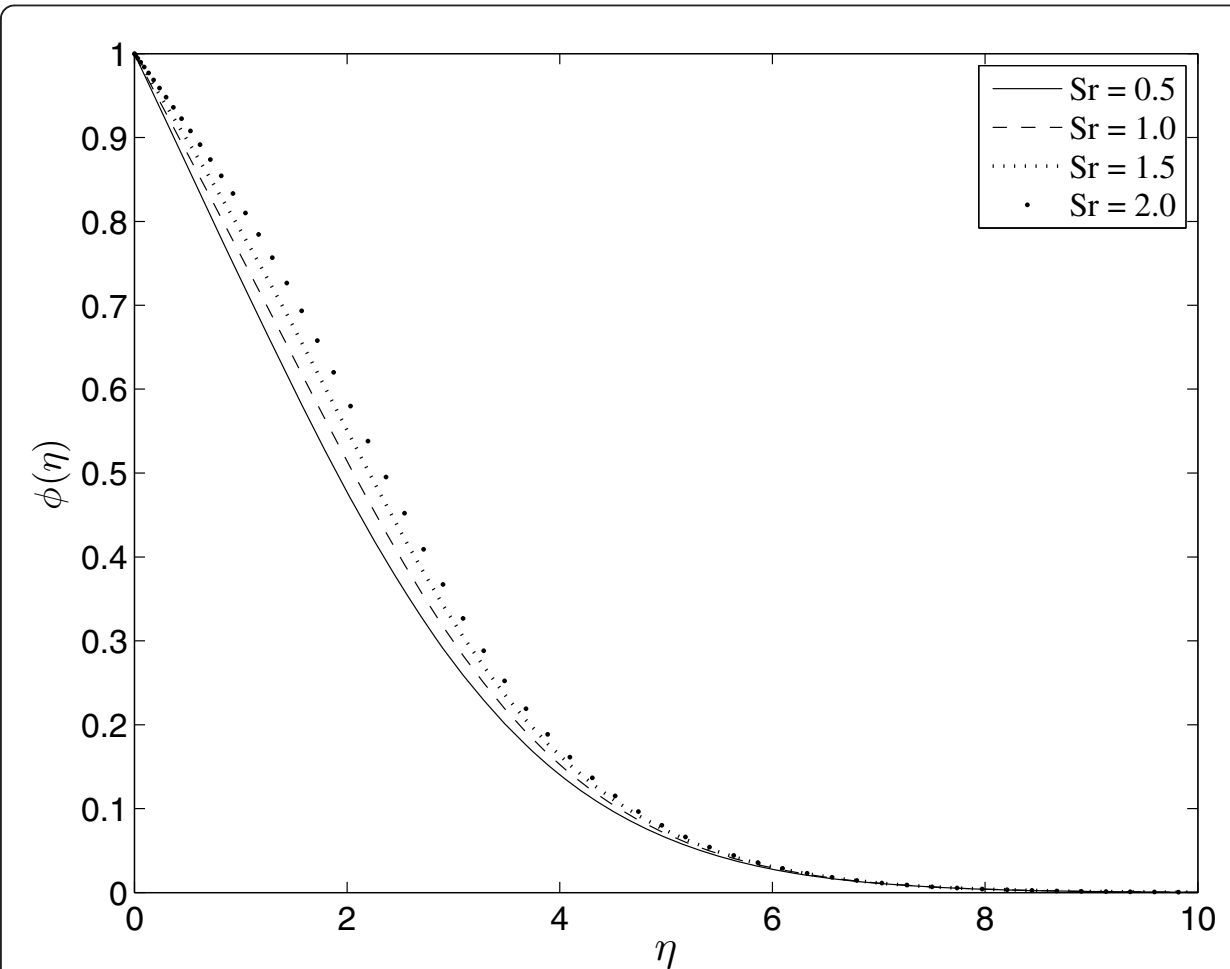

Figure 7 Effect of Soret parameter $\operatorname{Sr}$ on $\varphi(\eta)$ when $G r_{x}=0.1, G c_{x}=2.5, H a_{x}=0.1$, and $\xi=0.01$ 
Figure 5 shows the effect of increasing the Soret parameter (reducing the Dufour parameter) on the fluid velocity $f(\eta)$. The fluid velocity is found to increase with the Soret parameter.

The effect of Soret parameter on the temperature within the thermal boundary layer and the solute concentration is shown in Figures 6 and 7, respectively. An increase in the Soret effect reduces the temperature within the thermal boundary layer leading to an increase in the temperature gradient at the wall and an increase in heat transfer rate at the wall. On the other hand, increasing the Soret effect increases the concentration distribution which reduces the concentration gradient at the wall. These results are similar to the earlier findings by El-Kaberir [31] and Alam and Rahman [32], although the latter studies were subject to injection/suction.

\section{Conclusions}

In this article, we have investigated MHD and cross-diffusion effects on double-diffusive convection from a vertical flat plate in a viscous incompressible fluid. Numerical approximations for the governing equations were found using a combination of a regular perturbation expansion and the successive linearization method. The solutions were validated by using a local similarity, non-similarity method. We determined the effects of various parameters on the fluid properties as well as on the skin-friction coefficient, the heat and the mass transfer rates. We have shown that the magnetic field parameter enhances the temperature and concentration distributions within the boundary layer. The effect of thermo-diffusion is to reduce the temperature and enhance the velocity and the concentration profiles. The diffusion-thermo effect enhances the velocity and temperature profiles while reducing the concentration distribution. The skin-friction, heat and mass transfer coefficients decrease with an increase in the magnetic field strength. The skin-friction and heat transfer coefficients increase whereas the mass transfer coefficient decreases with increasing Soret numbers.

Acknowledgements

The authors would like to thank the referees for helpful comments and suggestions. This study was supported by the National Research Foundation (NRF). AAK was supported by the government of Sudan.

\section{Authors' contributions}

The problem was formulated following discussions by all three authors. Numerical simulations were carried out by AAK and FGA while the discussion was authored principally by PS. All authors read and approved the final manuscript.

\section{Competing interests}

The authors declare that they have no competing interests.

Received: 18 October 2011 Accepted: 24 February 2012 Published: 24 February 2012

\section{References}

1. Stern, ME: The 'salt fountain' and thermohaline convection. Tellus. 12, 172-175 (1960). doi:10.1111/j.2153-3490.1960. tb01295.x

2. Nield, DA, Bejan, A: Convection in Porous Media. Springer Verlag, New York (1999)

3. Nield, DA: Onset of thermohaline convection in a porous medium. Water Resour Res. 4, 553-560 (1968). doi:10.1029/ WR004i003p00553

4. Baines, PG, Gill, AE: On thermohaline convection with linear gradients. J Fluid Mech. 37, 289-306 (1969). doi:10.1017/ S0022112069000553

5. Rudraiah, N, Srimani, PK, Friedrich, R: Finite amplitude convection in a two component fluid saturated porous layer. Int J Heat Mass Transfer. 25, 715-722 (1982). doi:10.1016/0017-9310(82)90177-6

6. Poulikakos, D: Double diffusive convection in a horizontally sparsely packed porous layer. Int Commun Heat Mass Transfer. 13, 587-598 (1986). doi:10.1016/0735-1933(86)90035-7

7. Bejan, A, Khair, KR: Heat and mass transfer by natural convection in porous medium. Int J Heat Mass Transfer. 28, 909-918 (1985). doi:10.1016/0017-9310(85)90272-8 
8. Lai, FC: Coupled heat and mass transfer by natural convection from a horizontal line source in saturated porous medium. Int Commun Heat Mass Transfer. 17, 489-499 (1990). doi:10.1016/0735-1933(90)90067-T

9. Afify, AA: MHD free convective flow and mass transfer over a stretching sheet with chemical reaction. Heat Mass Transfer. 40, 495-500 (2004)

10. Makinde, OD, Sibanda, P: MHD mixed-convective flow and heat and mass transfer past a vertical plate in a porous medium with constant wall suction. J Heat Transfer. 130, 1-8 (2008)

11. Eckert, ERG, Drake, RM: Analysis of heat and mass transfer. McGraw-Hill, New York (1972)

12. Mortimer, RG, Eyring, H: Elementary transition state theory of the Soret and Dufour effects. Proc Natl Acad Sci. 77, 1728-1731 (1980). doi:10.1073/pnas.77.4.1728

13. Anjalidevi, SP, Devi, RU: Soret and Dufour effects on MHD slip flow with thermal radiation over a porous rotating infnite disk. Commun Nonlinear Sci. 16, 1917-930 (2011). doi:10.1016/j.cnsns.2010.08.020

14. Kafoussias, NG, Williams, EW: Thermal-diffusion and diffusion thermo effects on mixed free-forced convective and mass transfer boundary layer flow with temperature dependent viscosity. Int J Eng Sci. 33, 1369-1384 (1995). doi:10.1016/ 0020-7225(94)00132-4

15. Postelnicu, A: Influence of a magnetic field on heat and mass transfer by natural convection from vertical surfaces in porous media considering Soret and Dufour effects. Int J Heat Mass Transfer. 47, 1467-1472 (2004). doi:10.1016/j. ijheatmasstransfer.2003.09.017

16. Mansour, MA, El-Anssary, NF, Aly, AM: Effects of chemical reaction and thermal stratification on MHD free convective heat and mass transfer over a vertical stretching surface embedded in a porous media considering Soret and Dufour numbers. Chem Eng J. 145, 340-345 (2008). doi:10.1016/j.cej.2008.08.016

17. Lakshmi-Narayana, PA, Sibanda, P: Soret and Dufour effects on free convection along a vertical wavy surface in a fluid saturated Darcy porous medium. Int J Heat Mass Transfer. 53, 3030-3034 (2010). doi:10.1016/j. ijheatmasstransfer.2010.03.025

18. Awad, FG, Sibanda, P, Motsa, SS: On the linear stability analysis of a Maxwell fluid with double-diffusive convection. Appl Math Model. 34, 3509-3517 (2010). doi:10.1016/j.apm.2010.02.038

19. Liao, SJ: Beyond Perturbation: Introduction to the Homotopy Analysis Method. CRC Press, Boca Raton (2003)

20. Geng, F: Modified variational iteration method for second order initial value problems. Appl Appl Math. 1, 73-81 (2010)

21. Motsa, SS, Sibanda, P, Shateyi, S: A new spectral-homotopy analysis method for solving a nonlinear second order BVP. Commun Nonlinear Sci Numer Simulat. 15, 2293-2302 (2010). doi:10.1016/j.cnsns.2009.09.019

22. Motsa, SS, Sibanda, P, Awad, FG, Shateyi, S: A new spectral-homotopy analysis method for the MJD Jeffery-Hamel problem. Comput. Fluids. 39, 1219-1225 (2010). doi:10.1016/j.compfluid.2010.03.004

23. Makukula, ZG, Sibanda, P, Motsa, SS: A note on the solution of the Von Karman equations using series and chebyshev spectral methods. pp. 17. Boundary Value Problems2010, (2010) Article ID 471793

24. Makukula, ZG, Sibanda, P, Motsa, SS: A novel numerical technique for two-dimensional laminar flow between two moving porous walls. Math Problems Eng 2010, 15 (2010). Article ID 528956

25. Awad, FG, Sibanda, P, Motsa, SS, Makinde, OD: Convection from an inverted cone in a porous medium with crossdiffusion effects. Comput Math Appl. 61, 1431-1441 (2011). doi:10.1016/j.camwa.2011.01.015

26. Motsa, SS, Sibanda, P, Shateyi, S: On a new quasi-linearization method for systems of nonlinear boundary value problems. Math Methods Appl Sci. 34, 1406-1413 (2011). doi:10.1002/mma.1449

27. Afify, AA: Similarity solution MHD: Effects of thermal and diffusion thermo on free convective heat and mass transfer over a stretching surface considering suction or injection. Commun. Nonlinear Sci Numer Simulat. 14, 2202-2214 (2009). doi:10.1016/j.cnsns.2008.07.001

28. Sparrow, EM, Yu, HS: Local non-similarity thermal boundary layer solutions. J Heat Transfer. 93, 328-334 (1971). doi:10.1115/1.3449827

29. Sparrow, EM, Quack, H, Boerner, CJ: Local non-similarity boundary layer solutions. AIAA. 8, 1936-1942 (1970). doi:10.2514/3.6029

30. Shampine, LF, Ketzscher, R, Forth, SA: Using AD to solve BVPs in Matlab. ACM Trans Math Softw. 31(1):79-94 (2005) doi:10.1145/1055531.1055535

31. El-Kabeir, SMM: Soret and Dufour effects on heat and mass transfer by mixed convection over a vertical surface saturated porous medium with temperature dependent viscosity. Int J Numer Meth Fluids. (2011)

32. Alam, MS, Rahman, MM: Dufour and Soret effects on mixed convection flow past a vertical porous flat plate with variable suction. Nonlinear Anal: Model Control. 11, 3-12 (2006)

doi:10.1186/1687-2770-2012-25

Cite this article as: Sibanda et al:: On cross-diffusion effects on flow over a vertical surface using a linearization method. Boundary Value Problems 2012 2012:25. 